\title{
Bioactive versus Bare Platinum Coils in the Treatment of Intracranial Aneurysms: The MAPS (Matrix and Platinum Science) Trial
}

C.G. McDougall, S. Claiborne Johnston, A. Gholkar, S.L. Barnwell, J.C. Vazquez Suarez, J. Massó Romero, J.C. Chaloupka, A. Bonafe, A.K. Wakhloo, D. Tampieri, C.F. Dowd, A.J. Fox, S.J. Imm, K. Carroll, and A.S. Turk, for the MAPS Investigators

\begin{abstract}
BACKGROUND AND PURPOSE: The ability of polymer-modified coils to promote stable aneurysm occlusion after endovascular treatment is not well-documented. Angiographic aneurysm recurrence is widely used as a surrogate for treatment failure, but studies documenting the correlation of angiographic recurrence with clinical failure are limited. This trial compares the effectiveness of Matrix ${ }^{2}$ polyglycolic/polylactic acid biopolymer-modified coils with bare metal coils and correlates the angiographic findings with clinical failure (ie, target aneurysm recurrence), a composite end point that includes any incident of posttreatment aneurysm rupture, retreatment, or unexplained death.
\end{abstract}

MATERIALS AND METHODS: This was a multicenter randomized noninferiority trial with blinded end point adjudication. We enrolled 626 patients, divided between Matrix ${ }^{2}$ and bare metal coil groups. The primary outcome was target aneurysm recurrence at $12 \pm 3$ months.

RESULTS: At 455 days, at least 1 target aneurysm recurrence event had occurred in $14.6 \%$ of patients treated with bare metal coils and $13.3 \%$ of Matrix ${ }^{2}(P=.76$, log-rank test) patients; $92.8 \%$ of target aneurysm recurrence events were re-interventions for aneurysms that had not bled after treatment, and $5.8 \%$ of target aneurysm recurrence events resulted from hemorrhage or rehemorrhage, with or without retreatment. Symptomatic re-intervention occurred in only $4(0.6 \%)$ patients. At 455 days, $95.8 \%$ of patients with unruptured aneurysms and $90.4 \%$ of those with ruptured aneurysms were independent (mRS $\leq 2$ ). Target aneurysm recurrence was associated with incomplete initial angiographic aneurysm obliteration, presentation with rupture, and a larger aneurysmal dome and neck size.

CONCLUSIONS: Tested Matrix ${ }^{2}$ coils were not inferior to bare metal coils. Endovascular coiling of intracranial aneurysms was safe, and the rate of technical success was high. Target aneurysm recurrence is a promising clinical outcome measure that correlates well with established angiographic measurements.

ABBREVIATIONS: $B M C=$ bare metal coil; HELPS $=$ HydroCoil Endovascular Aneurysm Occlusion and Packing Study; ISAT $=$ International Subarachnoid Aneurysm Trial; MAPS $=$ Matrix and Platinum Science; TAR $=$ target aneurysm recurrence

$\mathbf{T}$ reatment of ruptured intracranial aneurysms with endovascular coiling is widely accepted, but incomplete or impermanent aneurysm occlusions are common. ${ }^{1,2}$ Although failure to achieve durable angiographic occlusion is frequent, delayed hemorrhage after coiling is infrequent. ${ }^{3}$ Because large sample sizes are

Received June 5, 2013; accepted after revision October 2.

From the Department of Neurosurgery (C.G.M.), Barrow Neurological Institute, St. Joseph's Hospital and Medical Center, Phoenix, Arizona; Clinical and Translational Science Institute (S.C.J.) and Department of Radiology (C.F.D.), University of California, San Francisco, San Francisco, California; Department of Neuroradiology (A.G.), Regional Neurosciences Center, Royal Victoria Infirmary, Newcastle Upon Tyne, UK; Departments of Neurological Surgery and Diagnostic Radiology (S.L.B.), Oregon Health and Science University, Portland, Oregon; Therapeutic Neuroradiology Unit (I.C.V.S.), University General Hospital of Alicante, Alicante, Spain; Department of Interventional Neuroradiology (I.M.R.), Hospital Donostia, San Sebastián, Spain; Department of Neurosurgery and Radiology (I.C.C.), Mount Sinai Medical Center, Miami Beach, Florida; Service de Neuroradiologie (A.B.), Hôspital Gui de Chauliac, Montpellier Cedex, France; Division of Neuroimaging and Intervention (A.K.W.), Department of Radiology, University of Massachusetts Medical School, Worcester, Massachusetts; Department of Diagnostic and Interventional Neuroradiology (D.T.), Montreal Neurological Institute, Montreal, Canada; Department of Neuroradiology (A.J.F.), Sunnybrook Health Sciences Center, Toronto, Ontario, Canada; Stryker Corporation (S.J.I., K.C.), Fremont, California; and

required to detect treatment effects on infrequent events, many trials use angiographic aneurysm residuals and/or recurrence as a surrogate for the much less frequent outcome of clinical failure, even though the correlation between angiographic failure and clinical failure is not well-characterized. While hemorrhage after treatment is the most concerning form of clinical failure, retreatment is also a significant negative clinical event for the patient

Departments of Neurointerventional Surgery, Radiology, and Neurosurgery (A.S.T.) Medical University of South Carolina, Charleston, South Carolina.

This work was sponsored by Stryker Corporation (Formerly Boston Scientific Corporation). Clinical Trial Registration-URL is http://www.clinicaltrials.gov/ ct2/show/NCT00396981?term = MAPS\&rank $=3$. The unique identifier is NCT00396981.

Please address correspondence to Cameron G. McDougall, MD, c/o Neuroscience Publications; Barrow Neurological Institute, St. Joseph's Hospital and Medical Center, 350 W. Thomas Rd, Phoenix, Arizona 85013; e-mail: neuropub@dignityhealth.org

三 Indicates article with supplemental on-line tables.

Indicates article with supplemental on-line figure.

Evidence-Based Medicine Level 1.

http://dx.doi.org/10.3174/ajnr.A3857 
and, as such, requires consideration. From the patient's perspective, it is the clinical, not the angiographic, outcome that is important, and it is, therefore, critical that the relationship between imaging and clinical outcomes be better understood.

Several varieties of polymer-modified coils have been developed with the goal of achieving more durable aneurysm occlusion. Matrix ${ }^{2}$ (Stryker, Kalamzoo, Michigan) is a platinum coil modified with a polyglycolic/polylactic acid braid. Although multiple studies of this coil and of a previous iteration of this coil have been conducted, ${ }^{4-19}$ this trial was initiated because no randomized trial had been conducted and the benefits of Matrix ${ }^{2}$ remained unproven.

The goals of this study were to compare Matrix ${ }^{2}$ with its bare metal counterpart the Guglielmi detachable coil (Stryker) and to use this trial to validate a composite clinical outcome measure created for the trial. The clinical outcome was designated as "target aneurysm recurrence" (TAR) and was defined as occurring when a patient experienced any of the following conditions after his or her initial aneurysm coiling:

1) Target aneurysm hemorrhage

2) Target aneurysm retreatment

3)Death from unknown cause.

\section{MATERIALS AND METHODS}

This randomized multicenter trial was conceived and designed by the investigators, with advice provided by the sponsor. This study was approved by all local institutional review boards. The study was conducted in accordance with the relevant parts of the International Conference on Harmonization Good Clinical Practice: Consolidated Guideline; the Declaration of Helsinki, EN ISO14155 Clinical Investigations of Medical Devices for Human Subjects; and the applicable regulations from the US Food and Drug Administration. Study centers were invited to participate; high-volume centers with broad geographic representation were selected for invitation.

\section{Study Subjects}

The study population included subjects $18-80$ years of age with a single untreated, intracranial saccular aneurysm (4-20 mm; Hunt and Hess scale score, I-III; mRS score, $0-3$ ), ruptured or unruptured, for which both polymer-modified coils and bare metal coils (BMCs) were treatment options and for which primary coiling treatment was planned to be completed during a single procedure.

Twenty-six of the 43 investigational sites were located in the United States. Due to the wide variability in the rate of patient recruitment among centers, large-volume centers were closed to enrollment after 60 patients were recruited to avoid having the recruitment dominated by a small number of large-volume centers. On-line Table 1 details enrollment by participating centers. Subjects were seen at the time of treatment and again $12 \pm 3$ months after the procedure. Preprocedural neurologic scores consisted of an independent assessment of the mRS score for all subjects plus a Hunt and Hess scale assessment for subjects with ruptured aneurysms. Long-term follow-up is to occur by telephone annually to 5 years post-index procedure.

All enrolled Matrix and Platinum Science (MAPS) Trial subject data are included in the intent-to-treat analysis except for 4 excluded subjects. One subject did not have an aneurysm, and 3 subjects were excluded at the request of the local institutional review board due to noncompliance with good clinical practice in obtaining informed consent. Overall, 626 MAPS Trial subjects were included in the intent-to-treat analyses.

\section{Procedures}

Patients were randomized in blocks of 2 and 4, stratified by target aneurysm rupture status and hospital site, to ensure equal distribution of those elements between the trial arms. Patients randomized to Matrix ${ }^{2}$ were to be treated with $\geq 75 \%$ total length of coils composed of Matrix ${ }^{2}$ and the remainder, of BMCs, while those randomized to BMCs were to be treated entirely with BMCs. To minimize confounding variables related to mechanical properties of different coil types, we allowed no other coil types or coils by different manufacturers in the index procedure. The use of adjunctive devices, specifically the use of the Neuroform stent (Stryker Neurovascular, Fremont, California), was at the operators' discretion.

As defined above, the primary outcome measure was TAR. Secondary outcome measures, all defined a priori, included angiographic assessment by site and core laboratory; assessments of $\mathrm{mRS}$ scores at $12 \pm 3$ months and direction of change from baseline, whether it improved or worsened, performed in person by an independent certified practitioner at a scheduled clinic visit; and technical procedural success, defined as the successful delivery and deployment of coils in the target aneurysm. Target aneurysm reintervention was defined as any further treatment of the aneurysm with the retreatment decision being at the discretion of the operator.

All sites graded their own angiographic outcomes on the basis of the modified 3-point Raymond scale postprocedurally and at followup. All sites also recorded an assessment of perceived change from baseline (same, better, worse) at follow-up. Digitized copies of the angiograms were created for all cases and were stored at an independent angiographic core laboratory located at the University of California, San Francisco. The core laboratory assessed all treatment and 1-year follow-up angiograms blinded to the treatment technique. Core laboratory evaluations were performed by using the same angiographic scales as the self-assessed outcomes.

An independent steering committee was responsible for overall oversight of the science and execution of the trial. Patient safety data were reviewed at regular intervals by an independent DataMonitoring Committee. An independent Clinical Events Committee was responsible for reviewing and adjudicating all deaths and neurologic events. Monitoring and source document verification were performed by an external monitoring clinical research organization, Geelen and Geelen. On-site monitoring and source document verification of Case Report Forms against original patient records were completed for $>40 \%$ of patients at the completion of the 1-year follow-up.

\section{Statistical Methods}

This study used a noninferiority design to evaluate whether Matrix $^{2}$ coils are noninferior to BMCs, with a clinically acceptable noninferiority margin set at $10 \%$, at a 1-sided significance level of $\alpha=.05$. Noninferiority was used to establish the baseline estimate from which future superiority studies could be conducted. Based on a literature review, the estimated 1-year TAR rate for aneurysms treated with BMCs was $20 \%$. Powering to noninferiority required 


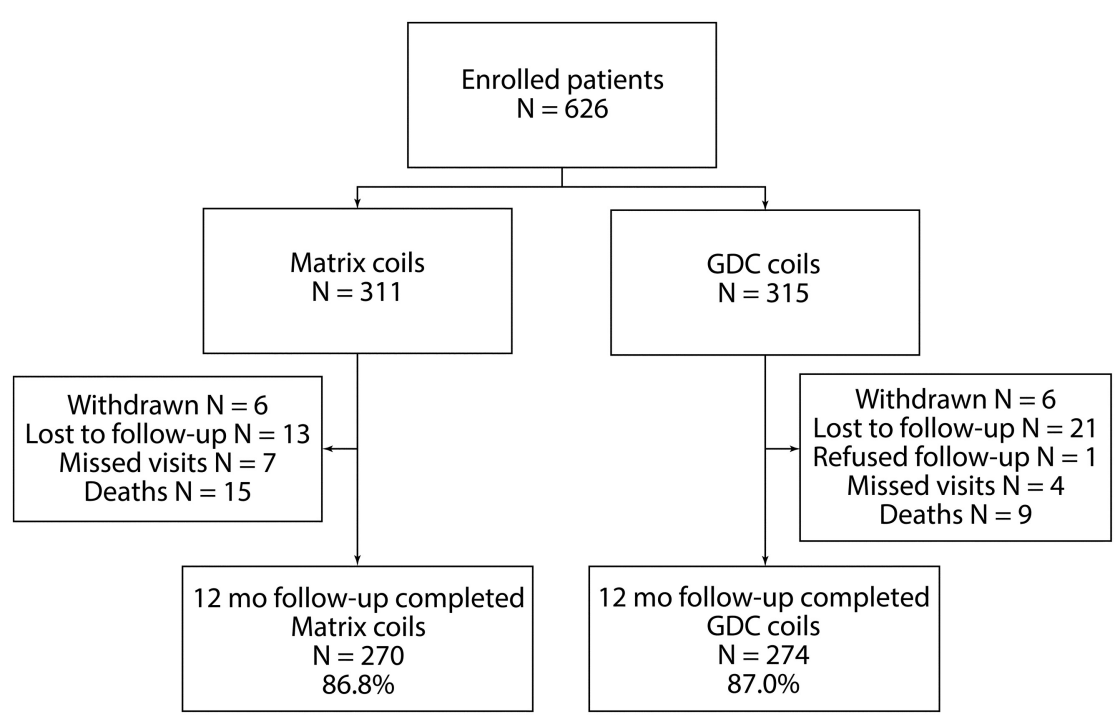

FIG 1. Subject flow through 455 days.

250 patients in each arm, with $87 \%$ power if the Matrix ${ }^{2}$ was equivalent to the BMC. Assuming 20\% lost to follow-up increased the sample size to 315 patients in each arm.

The primary end point (TAR) rate was calculated by using Kaplan-Meier estimates in each group at the end of a window of $12 \pm 3$ months (455 days). Time to event was based on the real time to rupture/rerupture, retreatment, or unknown cause of death, whichever happened first for each subject. Subjects who had not experienced an event were censored at their last clinical visit or 455 days, whichever came earlier. Noninferiority was to be claimed if the upper limit of the 1-sided 95\% confidence interval of the treatment difference was less than the prespecified $10 \%$ noninferiority margin. After showing noninferiority, a test for superiority was also to be conducted. Superiority was to be claimed if the upper limit of the 2 -sided $95 \%$ confidence interval of the treatment difference was $<0$.

The protocol prespecified additional subgroup analyses of TAR, modified Raymond scale, changes in mRS score from baseline to the 12-month assessment, and univariate and multivariate regression models to analyze TAR.

Logistic regression models were fitted to find baseline predictors for TAR, including baseline clinical and angiographic findings. The stepwise method was used with $P=.1$ entry and exit criteria for model selection.

A Student $t$ test was used for distributions of continuous variables between the groups. Either the $\chi^{2}$ or Fisher exact test was used to analyze binary variables according to standard statistical practice. For ordinal variables, such as the modified Raymond Scale, recanalization, and mRS scores, the Wilcoxon rank sum test was used to test the distribution between the groups. The differences between the groups were presented with the $95 \%$ confidence interval estimated by the normal approximation. For the binary outcomes, the relative risks as well as the $95 \%$ confidence intervals were also presented.

All statistical analyses were performed by using SAS, Version 9.2 (SAS Institute, Cary, North Carolina).

\section{RESULTS}

Between March 29, 2007, and October 20, 2009, six hundred twenty-six patients were enrolled in the MAPS trial (Fig 1). Thirty-three subjects received nonstudy coils, received no coils, or experienced a violation of the prespecified ratio of coil length. Nine subjects received excluded stents in addition to the randomized coil type. All 626 enrolled subjects were included in this intentto-treat analysis.

\section{Baseline Characteristics}

Baseline demographics were relatively evenly distributed between the 2 groups (Table 1). More patients had diabetes $(P=.0401)$ and a worse neurologic status at baseline in the Matrix ${ }^{2}$ arm than in the BMC arm. BMC patients with unruptured aneurysms had worse mRS scores $(P=.0457)$, and BMC patients with ruptured aneurysms had poorer Hunt and Hess scale scores $(P=.0136)$. Approximately $87 \%$ (543/626) of aneurysms treated were located in the anterior circulation; the most common location was the anterior communicating artery (On-line Table 2). There were no differences among aneurysm locations based on treatment group (On-line Table 2). Of the 228 (36\%) subjects who presented with acutely ruptured aneurysms, 218 (96\%) were treated within 14 days of rupture. Of the 626 subjects enrolled in the study, 509 (81\%) had wide-neck aneurysms based on study site measurements. The use of stents in wide-neck aneurysms was evenly distributed in both arms.

\section{Procedural Outcomes}

The procedural success rate was high in both treatment arms (97.5\% [307/315] for BMCs versus 96.8\% [301/311] for Matrix ${ }^{2}$ ). The inability to access the target aneurysm was the most common reason for technical failure. The rate of device malfunction was low in both groups (3.9\% [12/308] for BMCs versus 3.9\% [12/ 309] for Matrix ${ }^{2}$; On-line Table 3 ). The periprocedural complication rates (15.0\% [47/313] for BMCs versus 14.8\% [46/311] for Matrix $^{2}$ ) were consistent with other reports. ${ }^{13}$ On average, 6.9 coils were implanted per patient in the BMC arm compared with 5.5 coils in the Matrix ${ }^{2}$ arm. The packing attenuation of Matrix ${ }^{2}$ (26.4\%) was significantly greater $(P=.0013)$ compared with that of BMCs $(23.3 \%)$. Although the protocol allowed up to $25 \%$ of coil length in the Matrix ${ }^{2}$ arm to be BMCs, $71.1 \%$ (221/311) of patients in the Matrix ${ }^{2}$ arm received $100 \%$ Matrix $^{2}$.

\section{Primary End Point}

The Kaplan-Meier estimates of TAR at 455 days were $13.3 \%$ for the Matrix ${ }^{2}$ treatment group and $14.6 \%$ for BMC group (Fig 2). Noninferiority was shown with the upper limit (3.9\%) of a 1 -sided $95 \%$ confidence interval of the difference that was less than the prespecified $10 \%$ margin. However, the difference in TAR rates was not large enough to demonstrate superiority: The upper limit $(4.9 \%)$ of a 2 -sided $95 \%$ confidence interval of the 
Table 1: Summary of subject baseline characteristics ${ }^{\mathrm{a}}$

\begin{tabular}{lcc}
\hline \multicolumn{1}{c}{ Variable } & BMC $(\boldsymbol{n}=315)$ & Matrix $^{2}(\boldsymbol{n}=311)$ \\
\hline Male & $104(33.0 \%)$ & $82(26.4 \%)$ \\
Age (yr) & $54.4 \pm 13.2$ & $55.7 \pm 11.6$ \\
Ethnicity and race & & \\
$\quad$ Caucasian/white & $259(82.2 \%)$ & $249(80.1 \%)$ \\
Black or African American & $13(4.1 \%)$ & $12(3.9 \%)$ \\
Asian & $11(3.5 \%)$ & $13(4.2 \%)$ \\
Hispanic or Latino & $13(4.1 \%)$ & $16(5.1 \%)$ \\
Other & $19(6.0 \%)$ & $21(6.8 \%)$ \\
Current use of illicit drugs and/or alcohol abuse & $25(8.4 \%)$ & $25(8.6 \%)$ \\
Hypertension & $143(45.5 \%)$ & $153(49.5 \%)$ \\
Coronary disease & $29(9.4 \%)$ & $44(14.5 \%)$ \\
Hyperlipidemia/hypercholesterolemia & $70(22.5 \%)$ & $85(28.0 \%)$ \\
Intracranial atherosclerosis & $7(2.3 \%)$ & $4(1.3 \%)$ \\
Current smoking & $124(42.6 \%)$ & $117(41.6 \%)$ \\
Diabetes & $19(6.1 \%)$ & $33(10.7 \%)$ \\
Prior stroke or TIA & $35(11.2 \%)$ & $31(10.2 \%)$ \\
\hline
\end{tabular}

${ }^{a}$ Values are presented as mean \pm SD for continuous variables and No. (\%) for categoric variables. The denominator used for rates (\%) can be smaller than the number of subjects in each group due to missing values.

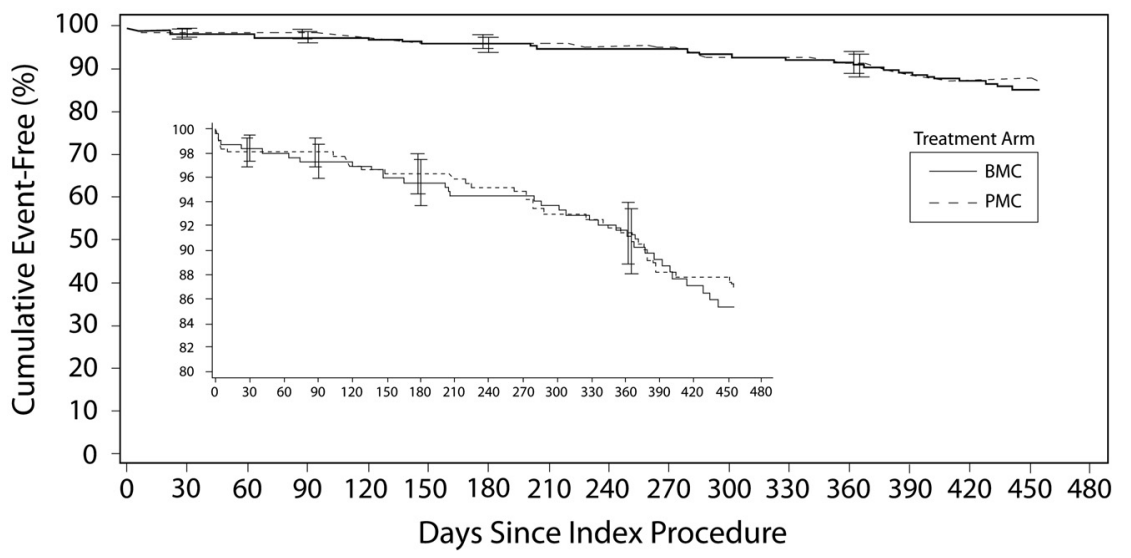

FIG 2. Kaplan-Meier curve showing freedom from TAR to 455 days in the intent-to-treat population $(n=626)$.

difference was $>0$. A Kaplan-Meier curve of per protocol analysis is shown in On-line Fig 1. Overall, 93\% (64/69) of TARs resulted from re-intervention of aneurysms that had not bled after treatment. Symptomatic re-intervention occurred in 4 of 626 patients ( $0.6 \%$, Table 2$), 3$ of whom had aneurysmal hemorrhage. One TAR event occurred due to an unexplained death. Subgroup analysis of the primary end point is given in On-line Table 4.

\section{Secondary End Points}

The immediate postprocedural angiographic assessment was similar between the 2 coil groups ( $P=.9894$, Table 3$)$. There was no difference in angiographic outcomes at 12 months between the 2 groups $(P=.8297)$. There was no significant difference in the 2 groups with respect to changes in the immediate post-index treatment and 12-month neurologic assessments compared with the preprocedural assessments.

The immediate postprocedural independent neurologic assessment demonstrated that 91\% (524/579) of the subjects had the same or better mRS scores compared with their pretreatment neurologic assessment. The results were similar for both treatment groups $(P=.4220)$. There also was no difference between the treatment groups with respect to worsening mRS scores from before the procedure to the 12-month follow-up (11.7\% [33/ 281] for BMCs versus 9.9\% [28/284] for Matrix $\left.{ }^{2}, P=.4705\right)$.

\section{Principal Safety Outcomes}

The Clinical Events Committee-adjudicated principal safety outcomes up to 455 days including all deaths, strokes, and ruptures/reruptures are presented in On-line Table 5. During the study, there were 837 safety events reported: $279(33 \%)$ as serious adverse events and $558(67 \%)$ as nonserious adverse events. Four $(0.6 \%, 4 / 626)$ aneurysms, 2 in each arm, ruptured or reruptured within 455 days of the index treatment. Twentyfour deaths were reported post-index treatment through 455 days. No unanticipated adverse device events were identified during the study. No cases of hydrocephalus were associated with unruptured aneurysms in either arm.

For all causes of death at 30 days, 3 patients died in the BMC arm and 12 in the Matrix ${ }^{2}$ arm $(P=.0174$, On-line Table 5). At 30 days, there was 1 neurologic death in the BMC arm and 11 in the Matrix $^{2}$ arm $(P=.0033)$. By 12 months, 9 patients (5 with ruptured aneurysms, $56 \%)$ in the BMC arm had died compared with 15 (11 presented ruptured, $73 \%)$ in the Matrix ${ }^{2}$ arm $(P=.2002)$. At 12 months, there was 1 neurologic death in the BMC arm versus 13 in the Matrix ${ }^{2}$ $\operatorname{arm}(P=.0011)$. There was no significant difference in the nonneurologic deaths at any time point.

All neurologic deaths were independently reviewed by the Clinical Events Committee. Deaths of known cause, unrelated to the target aneurysm or to potential neurologic etiologies (eg, pancreatic cancer, colon cancer, liver failure), were counted as non-neurologic and were not further evaluated. Neurologic deaths were adjudicated as related or not to the underlying disease, the procedure, and/or the study device, with the probability of the relationship being noted.

Of the 13 neurologic deaths in the Matrix ${ }^{2}$ group, 7 were ruled the result of the presenting hemorrhage, unrelated to either the procedure or the device. One death of unknown cause was automatically adjudicated as a TAR event, with unknown deaths being conservatively defined in the protocol as neurologic in cause and related to the study device. Two additional deaths occurred in patients with ruptured aneurysms for whom it was adjudicated that the procedure and or device may have played a role in addition to the effects of the presenting hemorrhage, and 3 procedural complications in patients with unruptured aneurysms (1 procedure-related ischemic stroke and 2 procedural hemorrhages) led to deaths. By contrast, of the 9 deaths occurring in the bare coil group, only 1 was neurologic in nature and was adjudicated as related to the procedure and study device. 
Based on the Clinical Events Committee adjudication, there were no statistically significant differences in neurologic deaths related to the study device, index procedure, or both. At both 30 days and 12 months, significantly more neurologic deaths in the Matrix $^{2}$ arm were adjudicated as unrelated to the study device and index procedure $(P=.0147$ and $P=.0072$, respectively).

There were 14 site-reported intraprocedural perforations: 8 $(2.5 \%, 8 / 315)$ in the BMC arm and $6(1.9 \%, 6 / 311)$ in the Matrix ${ }^{2}$ arm $(P=.6056)$. None of the perforations resulted in death.

Table 2: Number of TAR events at 455 days

\begin{tabular}{lccc}
\hline \multicolumn{1}{c}{ Subjects } & BMC (No.) (\%) & $\begin{array}{c}\text { Matrix } \mathbf{~}^{\mathbf{( N o}} \text { (\%) } \\
\text { (No) }\end{array}$ & $\begin{array}{c}\text { All Subjects } \\
\text { (No.) (\%) }\end{array}$ \\
\hline Overall & 315 & 311 & 626 \\
Subjects who met primary end point & $35(11.1)$ & $34(10.9)$ & $69(11.0)$ \\
Re-intervention only & $33(10.5)$ & $31(10.0)$ & $64(10.2)$ \\
Ruptures or reruptures & $2(0.6)$ & $2(0.6)$ & $4(0.6)^{\mathrm{a}}$ \\
Unknown causes of death & 0 & $1(0.3)$ & $1(0.2)$ \\
Symptomatic TAR & $3(1.0)$ & $4(1.3)$ & $7(1.1)$ \\
Symptomatic retreatment & $2(0.6)$ & $2(0.6)$ & $4(0.6)$ \\
Unruptured & 196 & 202 & 398 \\
Subjects who met primary end point & $18(9.2)$ & $19(9.4)$ & $37(9.3)$ \\
Re-intervention only & $18(9.2)$ & $18(8.9)$ & $36(9.0)$ \\
Ruptures or reruptures & 0 & $1(0.5)$ & $1(0.3)^{\mathrm{b}}$ \\
Unknown causes of death & 0 & 0 & 0 \\
Symptomatic TAR & $1(0.5)$ & $2(1.0)$ & $3(0.8)$ \\
Symptomatic retreatment & $1(0.5)$ & $2(1.0)$ & $3(0.8)$ \\
Ruptured & 119 & 109 & 228 \\
Subjects who met primary end point & $17(14.3)$ & $15(13.8)$ & $32(14.0)$ \\
Re-intervention only & $15(12.6)$ & $13(11.9)$ & $28(12.3)$ \\
Ruptures or reruptures & $2(1.7)$ & $1(0.9)$ & $3(1.3)^{\mathrm{C}}$ \\
Unknown causes of death & 0 & $1(0.9)$ & $1(0.4)$ \\
Symptomatic TAR & $2(1.7)$ & $2(1.8)$ & $4(1.8)$ \\
Symptomatic retreatment & $1(0.8)$ & 0 & $1(0.4)$ \\
\hline
\end{tabular}

${ }^{a}$ Three patients (2 BMC, 1 Matrix ${ }^{2}$ ) with a rupture/rerupture also had a subsequent re-intervention.

${ }^{b}$ One patient (Matrix ${ }^{2}$ ) with a rupture also had a subsequent re-intervention.

${ }^{c}$ Two patients $(B M C)$ with a rerupture also had a subsequent re-intervention.

\section{Subgroup Analysis of the Primary End Point (TAR)}

There were no significant differences between the groups based on aneurysm location, dome size, neck width, dome-to-neck ratio, rupture status, flow orientation, or use of adjunctive devices.

\section{Association between Primary and Secondary End Points}

The angiographic characteristics at treatment were assessed as predictors of TAR (Table 4). Incomplete aneurysm obliteration immediately after treatment tended to be associated with a greater likelihood of TAR at 455 days. Multivariate modeling of 12month TAR predictors (Table 5) included the rupture status of the aneurysm, the dome and neck size of the aneurysm, and the initial quality of the aneurysm occlusion (Raymond 3 versus Raymond 1). Among patients whose aneurysms were more completely occluded at the time of initial treatment (Raymond 1 or 2), TAR rates were lower in the Matrix ${ }^{2}$-treated patients $(2.7 \%$, $4 / 147)$ than in the BMC-treated patients (9.6\%, 15/157) $(P=.01)$. However, for patients with residual dome filling after initial treatment (Raymond 3), the TAR rate for the Matrix ${ }^{2}$-treated patients tended to be higher $(24.2 \%, 22 / 91)$ than that observed in the BMC-treated patients $(16.1 \%, 15 / 93)(P=.17)$.

\section{DISCUSSION}

The MAPS Trial has shown Matrix ${ }^{2}$ to be noninferior to BMCs. The composite end point of TAR-aneurysm hemor-

\section{Table 3: Secondary end points ${ }^{a}$}

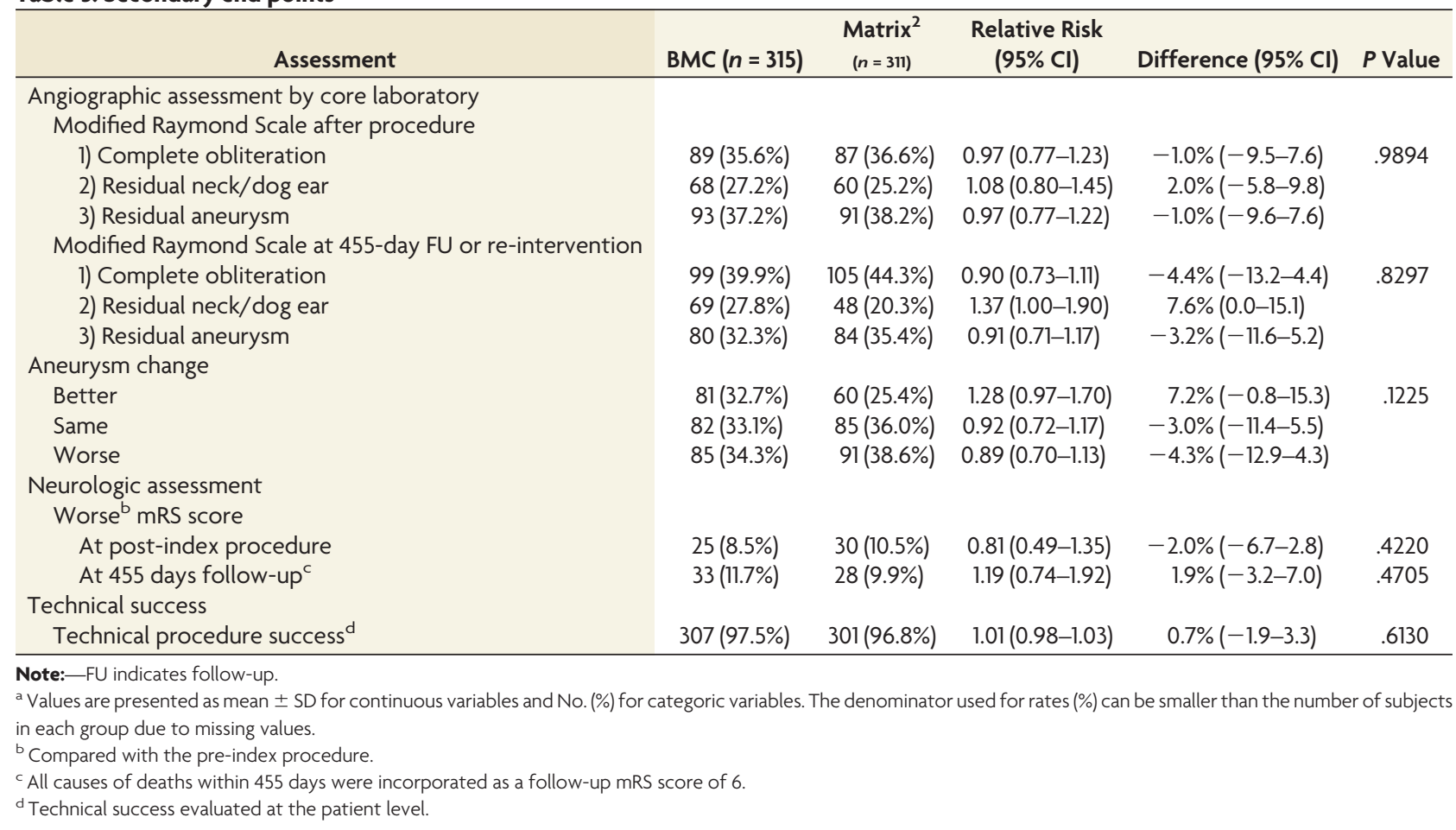


Table 4: 455-Day TAR rate by the Modified Raymond Scale at immediate postprocedure ${ }^{a}$

\begin{tabular}{lccccc}
\hline Modified Raymond Scale & BMC & Matrix & Relative Risk (95\% Cl) & Difference (95\% CI) & $P$ Value \\
\hline 1) Complete obliteration & $7.9 \%(7 / 89)$ & $3.4 \%(3 / 87)$ & $2.28(0.61-8.54)$ & $4.4 \%(-2.4-11.2)$ & .3297 \\
2) Residual neck/dog ear & $11.8 \%(8 / 68)$ & $1.7 \%(1 / 60)$ & $7.06(0.91-54.81)$ & $10.1 \%(1.8-18.4)$ \\
3) Residual aneurysm & $16.1 \%(15 / 93)$ & $24.2 \%(22 / 91)$ & $0.67(0.37-1.20)$ & $-8.0 \%(-19.6-3.5)$ & .1733 \\
\hline
\end{tabular}

${ }^{a}$ Values are presented as $\%(x / N)$.

Table 5: 455-Day TAR predictors by logistic regression models ${ }^{\mathrm{a}}$

\begin{tabular}{|c|c|c|c|c|}
\hline \multirow[b]{2}{*}{ Variable } & \multicolumn{3}{|c|}{ Standard } & \multirow[b]{2}{*}{$P$ Value } \\
\hline & Coefficient & Error & OR $(95 \% \mathrm{Cl})$ & \\
\hline Rupture status (ruptured vs unruptured) & 1.4 & 0.3 & $4.0(2.1-7.9)$ & $<.0001$ \\
\hline Core lab postproc Raymond Scale (2 vs 1) & 0.3 & 0.5 & $1.4(0.5-3.7)$ & .4917 \\
\hline Core lab postproc Raymond Scale (3 vs 1) & 1.6 & 0.4 & $5.0(2.2-11.0)$ & $<.0001$ \\
\hline Dome size $^{a}$ ( $\geq 10$ vs $<10 \mathrm{~mm}$ ) & 1.6 & 0.4 & $5.0(2.2-11.5)$ & .0001 \\
\hline Neck size ( $\geq 4$ vs $<4 \mathrm{~mm}$ ) & 0.8 & 0.3 & $2.3(1.2-4.4)$ & .0140 \\
\hline
\end{tabular}

Note:-postproc indicates postprocedural; AP, anteroposterior.

a Dome size is calculated as a minimum of the 2 widths (AP plane, lateral plane).

rhage after treatment, target aneurysm retreatment, and/or death from unknown cause-was reached in $13.3 \%$ of the Matrix ${ }^{2}$ treated patients and in $14.6 \%$ of the BMC-treated patients as Kaplan-Meier estimates at 455 days. While this study does not suggest any benefit from Matrix ${ }^{2}$ at 1 year, the 5-year follow-up results are not yet known.

Most of the TAR events were the result of asymptomatic target aneurysm retreatment. The significant baseline predictors of TAR included aneurysm size of $>10 \mathrm{~mm}$, neck size of $>4 \mathrm{~mm}$, pretreatment rupture status, and core laboratory adjudication of modified Raymond Scale 3 residual aneurysm filling on the immediate postprocedural angiogram. The immediate postprocedural Raymond Scale 3 versus 1 was a strong predictor of future TAR for Matrix ${ }^{2}$-treated patients (OR, 8.9; 95\% confidence interval, 2.6-31.1; $P=.0006)$ and tended to be so for the BMC-treated patients (OR, 2.3; 95\% confidence interval, $0.9-5.8 ; P=.0935)$ in a univariate fashion. Aneurysm retreatment strongly correlated with core laboratory adjudication of modified Raymond Scale 3 residual aneurysm filling at 455 days.

The primary end point of TAR is a practical, efficient, unequivocal end point that measures the clinical events that are important to patients. TAR can easily be applied in future trials and correlates well with angiographic benchmarks already in common use. Most TAR events were due to asymptomatic retreatments, given that the other elements of TAR (ie, hemorrhage and unexplained death) are relatively uncommon. Even though the association with angiographic aneurysm remnants and recurrent hemorrhage is not well-characterized, ${ }^{20}$ this poorly characterized threat drives most retreatments. In fact, 63 of 67 (94\%) retreatments occurred in asymptomatic subjects. By contrast, not all recurrences were retreated, again, not surprising, because the clinically important event of retreatment is based on additional factors beyond the angiographic findings. Retreatment cannot occur without residual aneurysm, but it is evident that treating physicians do not believe that all aneurysm residuals need to be treated. It is also likely that the clinicians making these retreatment decisions are selecting clinical and angiographic features that they believe place one patient at higher risk for hemorrhage than another.

The primary limitation of this study is that TAR most often resulted from the retreatment of asymptomatic angiographic an- eurysm remnants. This limitation naturally raises the question of whether TAR is a better outcome measure than core laboratory angiographic measurements. Additionally, although the core laboratory adjudicators were blinded to the coil type, the treating physicians were not. It can similarly be argued that the decision to retreat an aneurysm based on an asymptomatic angiographic recurrence is arbitrary. Indeed, different practitioners may have widely disparate thresholds for making the decision to retreat. While many residuals or recurrences may be innocuous, it is certain that some are not. Still, compared with an asymptomatic angiographic finding of residual aneurysm, retreatment is a much more important event to the patient, as are the other components of TAR. This relative importance of retreatment compared with purely angiographic end points is no less true even if the urgency of retreatment is uncertain and the risk is low. Additionally, while different practice patterns may make it difficult to compare TAR rates among trials, this difficulty does not diminish the applicability of TAR within a trial comparing 2 treatment modalities. With the 5-year follow-up that is ongoing in MAPS and additional long-term follow-up from other trials such as International Subarachnoid Aneurysm Trial (ISAT), whether an aggressive strategy of retreatment is warranted may become more apparent.

The impact of differing thresholds for retreatment is exemplified when MAPS is compared with the HydroCoil Endovascular Aneurysm Occlusion and Packing Study (HELPS) trial. Although the rates of major recurrence reported in the 2 "per protocol" treatment arms of HELPS were $24 \%$ and $34 \%$, respectively, the retreatment rate was only $3 \%$ in both groups. ${ }^{21}$ It is not clear why the retreatment rates in the 2 arms were the same despite a $10 \%$ difference in the rate of major recurrence between the 2 groups.

How well TAR correlates with angiographic outcomes and recurrent SAH will become clearer with the ongoing long-term follow-up occurring in MAPS and other trials. Although TAR correlated well with the angiographic occlusion scales, the core laboratory adjudication of immediate post-procedural occlusion correlated better with TAR at 455 days than did the local investigators' assessment. In $36 \%$ of cases, the local investigators reported a better degree of occlusion than did the core laboratory.

The correlation of TAR with the Raymond Scale of occlusion immediately after the procedure is a particularly useful finding. This correlation is important because the Raymond Scale is easily measured and is well-accepted. It is even more important, however, because unlike aneurysm size or rupture status, it is potentially modifiable at the time of initial aneurysm treatment. In other words, aggressive occlusion of the aneurysm at the first treatment is important in preventing a recurrence and in reducing 
the need for retreatment. Should long-term follow-up confirm a significant link between Raymond 3 aneurysm residuals and future hemorrhage, the clinical relevance of TAR will be enhanced.

The comparatively lower rate of TAR in the Matrix ${ }^{2}$-treated patients with an immediate Raymond 1 or 2 category occlusion is an interesting but inconclusive finding that requires replication. For patients with immediate posttreatment aneurysm occlusion scores of Raymond 1 or 2, the TAR rate was only $2.7 \%$. For patients with Raymond occlusion scores of 3, the TAR rate for Matrix $^{2}$ was higher than that seen in the BMC patients. This finding is biologically consistent with previous animal studies suggesting that an initially mechanically stable occlusion must be achieved to allow the biologic effect of clot stabilization to occur before the external polymer is absorbed.

The increased incidence of deaths in the Matrix ${ }^{2}$ arm was driven primarily by neurologic causes. It may be partially explained by differences in the comorbidities at the time of the index procedure (increased incidence of coronary artery disease, diabetes, and poor preprocedural neurologic status related to worse mRS or Hunt and Hess scale scores at admission). The much higher death rate in the Matrix ${ }^{2}$ patients was mostly the result of deaths adjudicated as unrelated to the study device or index procedure. However, 5 of the 13 deaths were adjudicated as being possibly related to the procedure and/or device, and 3 of these 5 deaths were in patients presenting with unruptured aneurysms.

An additional important finding of MAPS is that aneurysm coiling has become a remarkably safe procedure. Because of the sample size, a mix of patients with ruptured and unruptured aneurysms, prospective design, rigorous independent evaluations, and consistency with the findings of the HELPS and Cerecyte Coil Trials, ${ }^{21,22}$ important benchmarks with respect to the safety of endovascular aneurysm treatment are now confidently established. Regarding patients with ruptured aneurysms, at 455 days only 20 of 208 (9.6\%) were dead or disabled ( $\mathrm{mRS}>2$ ); this outcome compares favorably with the 1-year ISAT outcome of $23.5 \%$ by using the same outcome scale. ${ }^{1}$ Similarly, of the patients treated for unruptured aneurysms, only 15 of 360 patients (4.2\%) were dead or disabled at 455 days. This finding compares favorably with the International Study of Unruptured Intracranial Aneurysms, which reported (after excluding non-treatment-related deaths) a dead or disabled rate of $6.2 \%$ for the endovascular treatment of unruptured aneurysms. ${ }^{23}$ Both of these favorable comparisons are no doubt the result of improvements in technology and increased experience since these 2 landmark studies were completed.

More contemporary, higher quality comparisons are available. In HELPS, ${ }^{21}$ outcomes were adjudicated at 18 months. Poor outcomes $(\mathrm{mRS}>2)$ were seen in 24 of 214 patients $(11.2 \%)$ available for follow-up who had originally presented without recent hemorrhage and in 47 of 253 patients (18.6\%) who had originally presented with SAH. Most interesting, in the HELPS trial, only $3 \%$ of patients were retreated, though major angiographic recurrences were adjudicated in $27 \%-36 \%$ of the aneurysms at followup. Similar excellent procedural safety results have recently been reported in the Aneurysms Treated by Endovascular Approach registry and the Cerecyte Coil Trial. ${ }^{22,24}$ The Aneurysms Treated by Endovascular Approach study reported 1-month morbidity and mortality rates of $1.7 \%$ and $1.6 \%$, respectively. Likewise, the Cerecyte Coil Trial reported exceptionally good clinical outcomes in both treatment arms with only $2.7 \%$ of patients with unruptured aneurysms experiencing procedure-related neurologic deterioration.

Perhaps most reassuring in MAPS is that the posttreatment hemorrhage rate at 455 days was very low: 1 of 398 patients $(0.25 \%)$ for unruptured aneurysms and 3 of 228 patients (1.32\%) for previously ruptured aneurysms. These outcomes compare favorably with those in the ISAT trial, in which the rehemorrhage rate for aneurysms randomized to coiling after recent rupture was 28/1073 (2.6\%) within the first year after coiling, ${ }^{1}$ and with those in the Cerebral Aneurysm Rerupture After Treatment Trial, with its $3 \%$ rate at 1 year. $^{25}$

\section{CONCLUSIONS}

The MAPS Trial demonstrates that after 1 year of follow-up, Matrix $^{2}$ is not inferior to BMCs. Pending the results after 5 years of follow-up, in the absence of a finding of superiority for either coil type, cost and other factors may be important in choosing Matrix ${ }^{2}$ versus BMCs for clinical practice.

In addition, MAPS has shown TAR to be a practical clinical outcome measure that relates well to widely used angiographic assessments. Again, longer term follow-up should help clarify the utility of TAR as an outcome measurement.

Finally, MAPS provides additional evidence that coiling of intracranial aneurysms can be done with reliably excellent outcomes as evidenced by a very high rate of technical success, low treatment-related morbidity and mortality rates, and low posttreatment hemorrhage rates. These findings suggest continued improvement in the endovascular treatment of intracranial aneurysms, compared with earlier trials. ${ }^{9}$ The broad geographic nature of this multicenter trial and broad inclusion criteria suggest widespread generalizability of the study results.

Disclosures: Cameron G. McDougall—UNRELATED: Consultancy: Covidien, MicroVention, Comments: scientific advisory board for Covidien and proctor for ev3, consultant to MicroVention. S. Claiborne Johnston—RELATED: Grant: Stryker Neurovascular.* Anil Gholkar-RELATED: Grant: My hospital trust received a small grant for recruiting patients in the MAPS Trial. This was for collection of data for the study, * Support for Travel to Meetings for the Study or Other Purposes: Stryker, Comments: I received support for travel to investigator meetings, UNRELATED: Consultancy: Codman Neurovascular, Stryker Neurovascular, Johnson \& Johnson; Comments: I work as a consultant and was paid an honorarium for educational activities sponsored by device companies. The activities are around training of interventional neuroradiology trainees, Travel/Accommodations/Meeting Expenses Unrelated to Activities Listed: MicroVention, Comments: travel support to attend World Federation of Interventional and Therapeutic Neuroradiology and Society of Neurointerventional Surgery meetings from various device-manufacturing companies, Other: In the past, an interventional neuroradiology trainee at my hospital was partly supported by Stryker Neurovascular. As far as I am aware, this has not been the case for at least several years. * Stanley L. Barnwell—Consultancy: Stryker Neurovascular. Javier Massó Romero—RELATED: Grant: Stryker Neurovascular, ${ }^{*}$ Consulting Fee or Honorarium: Stryker Neurovascular. John C. Chaloupka-RELATED: Grant: Stryker Neurovascular, ${ }^{*}$ Comments: administrative support for data collection and follow-up for the trial, UNRELATED: Consultancy: Stryker Neurovascular, Comments: expert clinical and technical advice on various research and development projects, Payment for Lectures (including service on Speakers Bureaus): received payment from Stryker Neurovascular for sponsored lectures, Comments: educational lecturing on various topics involving the use of stenting in neuroendovascular practice. Alain Bonafe-RELATED: Grant: Stryker, ${ }^{\star}$ Support for Travel to Meetings for the Study or Other Purposes: Stryker, UNRELATED: Consultancy: Stryker. Ajay K. Wakhloo- UNRELATED: Board Membership: Surpass Medical Ltd, Consultancy: Stryker Neurovascular, Codman Johnson \& Johnson Neurovascular, Philips Health- 
care, Boston Biomedical Associates, Soteira, Expert Testimony: Hugsland and Knopf, LLC, New Jersey, Grants/Grants Pending: National Institutes of Health, ${ }^{*}$ Philips Healthcare, ${ }^{*}$ Payment for Lectures (including service on Speakers' Bureaus): Harvard postgraduate course, Stock/Stock Options: Stryker Neurovascular, Travel/Accommodations/Meeting Expenses Unrelated to Activities Listed: Stryker Neurovascular, Codman Johnson \& Johnson Neurovascular, Surpass Medical Ltd. Christopher F. Dowd-RELATED: Fees For Participation in Review Activities such as Data Monitoring Boards, Statistical Analysis, Endpoint Committees, and the Like: Stryker Neurovascular, Comments: I served as Chief Adjudicator for the MAPS Trial. I also served as a Medical Advisor to Neuro Vasx Inc. Allan J. Fox-RELATED: Grant: core laboratory for the Cerecyte Trial, Support for Travel to Meetings for the Study or Other Purposes: Micrus Endovascular, ${ }^{*}$ Comments: for meetings of Cerecyte; OTHER RELATIONSHIPS: Chair of Adverse Events Committee for the MAPS Trial, payment per hour for work done, organized by Stryker Neurovascular. So Jung Imm-RELAT$E D$ : I worked for Stryker Neurovascular, which sponsored this study. I did not receive compensation beyond my normal salary for working on this project. UNRELATED: Employment: Stryker Neurovascular, Comments: At the time of this study, I was an employee of Stryker Neurovascular. Kirsten Carroll—RELATED: Grant: I work for Stryker, which sponsored the study. I did not receive compensation beyond my normal salary for working on this project. I did not receive third-party funding outside of Stryker for my work on the project, UNRELATED: Employment: Stryker Neurovascular, Comments: I am an employee of Stryker Neurovascular; Stock/Stock Options: Stryker Neurovascular, Comments: I receive stock grants as part of my annual compensation at Stryker Neurovascular. Aquilla S. Turk-RELATED: Consulting Fee or Honorarium: Stryker, Comments: consultation work, honoraria, research funding from Stryker, UNRELATED: Consultancy: Covidien, MicroVention, Penumbra, Stryker (other projects), Siemens, Codman, Comments: consultation work, honoraria, research funding, Grants/Grants Pending: Medical University of South Carolina, ${ }^{*}$ Comments: for the clinical trial LARGE, Payment for Lectures (including service on Speakers Bureaus): Covidien, MicroVention, Penumbra, Stryker (other projects), Siemens, Codman. *Money paid to the institution.

\section{REFERENCES}

1. Molyneux AJ, Kerr RS, Yu LM, et al. International subarachnoid aneurysm trial (ISAT) of neurosurgical clipping versus endovascular coiling in 2143 patients with ruptured intracranial aneurysms: a randomised comparison of effects on survival, dependency, seizures, rebleeding, subgroups, and aneurysm occlusion. Lancet 2005;366:809-17

2. McDougall CG, Spetzler RF, Zabramski JM, et al. The Barrow Ruptured Aneurysm Trial. J Neurosurg 2012;116:135-44

3. Molyneux AJ, Kerr RS, Birks J, et al. Risk of recurrent subarachnoid haemorrhage, death, or dependence and standardised mortality ratios after clipping or coiling of an intracranial aneurysm in the International Subarachnoid Aneurysm Trial (ISAT): long-term follow-up. Lancet Neurol 2009;8:427-33

4. Kang HS, Han MH, Kwon BJ, et al. Short-term outcome of intracranial aneurysms treated with polyglycolic acid/lactide copolymercoated coils compared to historical controls treated with bare platinum coils: a single-center experience. AJNR Am J Neuroradiol 2005;26:1921-28

5. Taschner CA, Leclerc X, Rachdi H, et al. Matrix detachable coils for the endovascular treatment of intracranial aneurysms: analysis of early angiographic and clinical outcomes. Stroke 2005;36:2176-80

6. Lubicz B, Leclerc X, Gauvrit JY, et al. Endovascular treatment of intracranial aneurysms with Matrix coils: a preliminary study of immediate post-treatment results. AJNR Am J Neuroradiol 2005;26:373-75

7. Raymond J, Guilbert F, Weill A, et al. Long-term angiographic recurrences after selective endovascular treatment of aneurysms with detachable coils. Stroke 2003;34: 1398-1403

8. Heros RC, Zauner A. Early experience with Matrix detachable coils. J Neurosurg 2006;105:190-91, discussion 191

9. Murayama Y, Vinuela F, Ishii A, et al. Initial clinical experience with
Matrix detachable coils for the treatment of intracranial aneurysms. J Neurosurg 2006;105:192-99

10. Fiorella D, Albuquerque FC, McDougall CG. Durability of aneurysm embolization with Matrix detachable coils. Neurosurgery 2006;58: 51-59

11. Niimi Y, Song J, Madrid M, et al. Endosaccular treatment of intracranial aneurysms using Matrix coils: early experience and midterm follow-up. Stroke 2006;37:1028-32

12. Katsaridis V, Papagiannaki C, Violaris C. Guglielmi detachable coils versus Matrix coils: a comparison of the immediate posttreatment results of the embolization of 364 cerebral aneurysms in 307 patients-a single-center, single-surgeon experience. AJNR Am J Neuroradiol 2006;27:1841-48

13. Pierot L, Bonafe A, Bracard S, et al. Endovascular treatment of intracranial aneurysms with Matrix detachable coils: immediate posttreatment results from a prospective multicenter registry. AJNR Am J Neuroradiol 2006;27:1693-99

14. Rivet DJ, Moran CJ, Mazumdar A, et al. Single-institution experience with Matrix coils in the treatment of intracranial aneurysms: comparison with same-center outcomes with the use of platinum coils. AJNR Am J Neuroradiol 2007;28:1736-42

15. Pierot L, Leclerc X, Bonafe A, et al. Endovascular treatment of intracranial aneurysms using Matrix coils: short- and mid-term results in ruptured and unruptured aneurysms. Neurosurgery 2008;63:850-57

16. Pierot L, Leclerc X, Bonafe A, et al. Endovascular treatment of intracranial aneurysms with Matrix detachable coils: midterm anatomic follow-up from a prospective multicenter registry. AJNR Am J Neuroradiol 2008;29:57-61

17. Ishii A, Murayama Y, Nien YL, et al. Immediate and midterm outcomes of patients with cerebral aneurysms treated with Matrix1 and Matrix 2 coils: a comparative analysis based on a single-center experience in 250 consecutive cases. Neurosurgery 2008;63:1071-77

18. Smith MJ, Mascitelli J, Santillan A, et al. Bare platinum vs Matrix detachable coils for the endovascular treatment of intracranial aneurysms: a multivariate logistic regression analysis and review of the literature. Neurosurgery 2011;69:557-64

19. Pierot L, Cognard C, Ricolfi F, et al. Mid-term anatomic results after endovascular treatment of ruptured intracranial aneurysms with Guglielmi detachable coils and Matrix coils: analysis of the CLARITY series. AJNR Am J Neuroradiol 2012;33:469-73

20. Johnston SC, Dowd CF, Higashida RT, et al. Predictors of rehemorrhage after treatment of ruptured intracranial aneurysms: the Cerebral Aneurysm Rerupture After Treatment (CARAT) study. Stroke 2008;39:120-25

21. White PM, Lewis SC, Gholkar A, et al. Hydrogel-coated coils versus bare platinum coils for the endovascular treatment of intracranial aneurysms (HELPS): a randomised controlled trial. Lancet 2011;377:1655-62

22. Coley S, Sneade M, Clarke A, et al. Cerecyte Coil Trial: procedural safety and clinical outcomes in patients with ruptured and unruptured intracranial aneurysms. AJNR Am J Neuroradiol 2012;33:474-80

23. Wiebers DO, Whisnant JP, Huston J III, et al. Unruptured intracranial aneurysms: natural history, clinical outcome, and risks of surgical and endovascular treatment. Lancet 2003;362:103-10

24. Pierot L, Spelle L, Vitry F. Immediate clinical outcome of patients harboring unruptured intracranial aneurysms treated by endovascular approach: results of the ATENA study. Stroke 2008;39:2497-504

25. The CARAT Investigators. Rates of delayed rebleeding from intracranial aneurysms are low after surgical and endovascular treatment. Stroke 2006;37:1437-42 